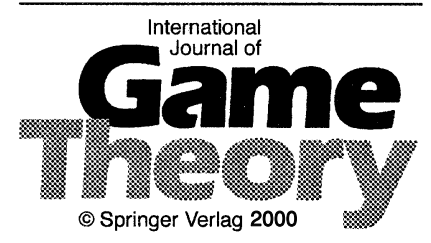

\title{
Communication, credible improvements and the core of an economy with asymmetric information
}

\author{
Oscar Volij ${ }^{1,2, *}$ \\ ${ }^{1}$ Economics Department, Brown University, Providence, Rhode Island 02912-B, USA \\ (e-mail: oscar_volij@brown.edu) \\ ${ }^{2}$ Department of Economics, The Hebrew University of Jerusalem, Jerusalem 91905, Israel
}

Received: September 1998/revised version: June 1999

\begin{abstract}
We analyze an economy with asymmetric information and endogenize the possibilities for information transmission between members of a coalition. We then define a concept of the Core that takes into account these communication possibilities. The internal consistency of the improvements is considered and an Internally Consistent Core, which requires credibility from the improvements is introduced.
\end{abstract}

Key words: Asymmetric information, core, common knowledge

\section{Introduction}

In his seminal paper Wilson (1978) analyzes an economy with asymmetric information and proposes a definition of the Core. The difficulty raised by economies with asymmetric information stems from two factors: first, agents evaluate bundles after they have received their information, and secondly, not all of them have the same information. If agents evaluated bundles before their private information is revealed to them or, if all agents received the same information, we would face a special case of Arrow-Debreu economies and need no new definition of the Core. Wilson (1978) notes that when information is asymmetric, it is not enough for each member of a coalition to know that he prefers one allocation over another in order for a coalition to improve upon the latter. It must be commonly known by all members of the coalition that this is so. The requirement that the improvement be common knowledge is needed because agents necessarily learn that they are improving upon an

* I thank Darin Lee, Roberto Serrano, Rajiv Vohra and especially Nir Dagan and Ronel Elul for illuminating discussions. 
allocation when they are doing so, and they must still be willing to transact after they have learned whatever they have learned. The important question is: With respect to what information structure should an improvement be common knowledge?

When there are no opportunities for communication, the proper response to the question above is: with respect to the agents' initial information. For if there is no information transmission, the agents cannot refine their initial information and consequently, a coalition can improve upon an allocation only if it is self-evident that they can enforce something better. Wilson's Coarse Core takes these circumstances into account. When opportunities for communication are allowed, on the other hand, the relevant information is the initial information refined by the information transmission that has taken place. Wilson's Fine Core takes this into account and allows for unlimited communication among agents.

The possibilities of communication are exogenously given in the definition of both the Coarse and the Fine core. In this paper we want to make the information transmission endogenous. The idea is that while it may not be common knowledge that a given allocation is strictly preferred by the members of some coalition, it may become common knowledge after all of them express their willingness to trade. That is, if agents are allowed to repeatedly express their willingness to carry out a trade, the common desirability of one allocation over another may become common knowledge. The information transmission is endogenous because it follows from the comparison of two given allocations, and different allocations will give rise to different information transmissions. The way the information is transmitted and common knowledge is thus achieved, is similar to the processes by which posteriors converge in Geanakoplos and Polemarchakis (1982) and in Bacharach (1985). ${ }^{1}$ When we allow for the kind of endogenous information transmission described above, we are lead to a definition of the Core that is intermediate between the Coarse Core and the Fine Core.

As Wilson (1978) notes, the opportunities for communication may disrupt arrangements for mutual insurance, resulting in the emptiness of the Core. This is true for both the Fine Core and for our Core with endogenous communication. But there is another disruption that the opportunities for communication may cause, and it is related to the credibility of the improvements. Specifically, there are allocations outside the Core with communication that cannot be improved upon in a credible way. Namely, whenever they are improved upon by a coalition $S$ by means of an allocation $y$ and after some information transmission has taken place, there is a subcoalition of $S$ that can use its new information and improve upon $y$ by means of some other assignment of bundles $z$. This problem raises the question of what the allocations that cannot be improved upon in an internally consistent way are. When the sort of endogenous information transmission described above is allowed, the answer is what we call the Internally Consistent Core.

The Internally Consistent Core clearly contains the Core with endogenous communication, but there is no inclusion relation with the Coarse Core. The fact that it is not a subset of the Coarse Core may be surprising at first sight,

\footnotetext{
${ }^{1}$ See also Sebenius and Geanakoplos (1983), and Hart and Tauman (1996) for an economic application.
} 
but it is a natural consequence of allowing communication. For example an allocation $y$ that is commonly known to be strictly preferred to another allocation $x$ when they are evaluated with the initial information may not be credible, because a subcoalition may improve upon the former using some communication which may be insufficient to improve upon the latter.

Although the Coarse Core, the Core with communication, and the Internally Consistent Core are different concepts, the improvements on which they are based essentially coincide when we restrict attention to the grand coalition and to one-person coalitions. This allows us to prove a modified version of the No Trade Theorem (see Milgrom and Stokey (1982) for an early version of the theorem).

In this paper, as in Wilson (1978), we abstract from incentive problems. Implicit in the definition of an allocation is the assumption that the states are verifiable. We do this because we want to focus on the topic of endogenous information transmission and the appropriate concept of improvements. For a paper that takes into account incentive problems and thus restricts attention to the allocations that are incentive compatible, see Vohra (1999). A more related paper is Dutta and Vohra (1997) which, while restricting attention to incentive compatible allocations, attempts to endogenize the information transmission. Holmstrom and Myerson (1983) and Krasa (1999) deal with endogenous communication, but they restrict attention to the grand coalition.

The paper is organized as follows. Section 2 presents the model of an economy with asymmetric information and the allocations that we consider. Section 3 discusses several notions of improving allocations, which differ only in the communication possibilities they allow. When the transmission of information is endogenous, the corresponding notion of improving allocations gives rise to the Core with endogenous communication. After noting that some improvements with endogenous communication may not be internally consistent, Section 4 uses the Core with endogenous communication as a building block to define the Internally Consistent Core, which is the main concept of the paper. The central message of the paper is conveyed in Example 3, which shows that belonging to the Coarse Core is not a necessary condition for stability when endogenous communication is allowed.

\section{Economies}

Let $\Omega$ be a finite set with generic element $\omega$. The set $\Omega$ represents the set of possible states of the world, and $\omega$ represents one such state. Subsets of $\Omega$ are called events. A commodity vector is an element of $\mathbb{R}_{+}^{l}$. A bundle is a function $x_{i}: \Omega \rightarrow \mathbb{R}_{+}^{l}$ that assigns a commodity vector to each state of the world. We denote the set of bundles by $\mathbb{B}$. An agent $i$ is a fourtuple $\left(\mathscr{P}_{i}, u_{i}, e_{i}, \mu_{i}\right)$ where

$\mathscr{P}_{i}$ is a partition of $\Omega$ that represents $i$ 's information

$u_{i}: \mathbb{R}_{+}^{l} \times \Omega \rightarrow \mathbb{R}$ is agent $i$ 's state contingent utility function

$e_{i}: \Omega \rightarrow \mathbb{R}_{+}^{l}$ is agent $i$ 's state contingent initial endowment of commodities

$\mu_{i}$ is a strictly positive probability measure on $\Omega$ that represents agent $i$ 's prior beliefs.

For each partition $\Pi$ of $\Omega$, we denote by $\Pi(\omega)$ the element of $\Pi$ that contains $\omega$. For each bundle $x_{i} \in \mathbb{B}$ we denote by $u_{i}\left(x_{i}\right)$ the function $u_{i}\left(x_{i}\right)$ : 
$\Omega \rightarrow \mathbb{R} \mid u_{i}\left(x_{i}\right)(\omega)=u_{i}\left(x_{i}(\omega), \omega\right)$. Let $\Pi_{i}$ be a partition of $\Omega$ and let $x_{i}: \Omega \rightarrow$ $\mathbb{R}$ be a bundle. Agent $i$ 's conditional expected utility of $x_{i}$ relative to $\Pi_{i}$ is the function $\mathrm{E}_{\mu_{i}}\left(u_{i}\left(x_{i}\right) \mid \Pi_{i}\right): \Omega \rightarrow \mathbb{R}$ that assigns to each state $\omega$, agent $i$ 's conditional expectation of $u_{i}\left(x_{i}\right)$ given the event $\Pi_{i}(\omega)$, namely

$$
\mathrm{E}_{\mu_{i}}\left(u_{i}\left(x_{i}\right) \mid \Pi_{i}\right)(\omega)=\mathrm{E}_{\mu_{i}}\left(u_{i}\left(x_{i}\right) \mid \Pi_{i}(\omega)\right) .
$$

Definition 1. An economy with asymmetric information $\mathscr{E}=$ $\left\langle N,\left(\mathscr{P}_{i}, u_{i}, e_{i}, \mu_{i}\right)_{i \in N}\right\rangle$, is a finite collection of agents.

Non-empty subsets of $N$ are called coalitions.

Definition 2. Let $S$ be a coalition. An information structure for $S$ is a collection $\left(\Pi_{i}\right)_{i \in S}$ of partitions of $\Omega$, one partition for each agent in $S$.

Information structures for $N$ are called simply information structures.

Let $\left(\Pi_{i}\right)_{i \in S}$ be an information structure for $S$. The meet of the partitions $\left(\Pi_{i}\right)_{i \in S}$ is the finest partition of $\Omega$ that is coarser than each $\Pi_{i}, i \in S$, and it is denoted by $\Pi_{S}=\bigwedge_{i \in S} \Pi_{i}$. Similarly, the join of the partitions $\left(\Pi_{i}\right)_{i \in S}$ is the coarsest partition that is finer than each $\Pi_{i}, i \in S$ and is denoted by $\bigvee_{i \in S} \Pi_{i}$. An event $E$ is said to be common knowledge among the members of $S$ at $\omega$ with respect to information structure $\left(\Pi_{i}\right)_{i \in S}$ if $\Pi_{S}(\omega) \subseteq E$. If an information structure is not specified, it should be understood that we mean the initial information partitions $\mathscr{P}_{i}$. The formal definition of common knowledge was first introduced by Aumann (1976).

Definition 3. Let $S$ be a coalition. An assignment of bundles to agents in $S$ is a function $y: S \rightarrow \mathbb{B}$.

Definition 4. Let $\mathscr{E}=\left\langle N,\left(\mathscr{P}_{i}, u_{i}, e_{i}, \mu_{i}\right)_{i \in N}\right\rangle$ be an economy and let $S$ be a coalition. An $S$-allocation is an assignment of bundles to agents in $S$ such that $\sum_{i \in S} y(i)=\sum_{i \in S} e_{i}$.

$S$-allocations represent all those redistributions of their endowments that coalition $S$ can carry out without the consent of others. $N$-allocations are simply called allocations. Denote the set of all $S$-allocations by $\mathscr{A}(S)$ and the set of allocations by $\mathscr{A}$. If $y$ is an assignment of bundles to agents in $S$ and $T \subseteq S$, we shall write $y_{T}$ for the projection of $y$ on $T$ and, with a slight abuse of notation, $y_{i}$ for $y(i)$.

Definition 5. Let $\mathscr{E}=\left\langle N,\left(\mathscr{P}_{i}, u_{i}, e_{i}, \mu_{i}\right)_{i \in N}\right\rangle$ be an economy. We say that allocation $x$ is individually rational if

$$
\mathrm{E}_{\mu_{i}}\left(u_{i}\left(x_{i}\right) \mid \mathscr{P}_{i}\right) \geq \mathrm{E}_{\mu_{i}}\left(u_{i}\left(e_{i}\right) \mid \mathscr{P}_{i}\right) \quad \forall i \in N .
$$

Definition 6. Let $\mathscr{E}=\left\langle N,\left(\mathscr{P}_{i}, u_{i}, e_{i}, \mu_{i}\right)_{i \in N}\right\rangle$ be an economy. We say that allocation $x$ is strictly efficient if there is no allocation $y \in \mathscr{A}$ and no state $\omega^{*} \in \Omega$ such that

$$
\mathrm{E}_{\mu_{i}}\left(u_{i}\left(y_{i}\right) \mid \mathscr{P}_{i}\right)(\omega) \geq \mathrm{E}_{\mu_{i}}\left(u_{i}\left(x_{i}\right) \mid \mathscr{P}_{i}\right)(\omega) \quad \forall \omega \in \mathscr{P}_{N}\left(\omega^{*}\right), \quad \forall i \in N,
$$

with strict inequality at $\omega^{*}$ for some $i \in N$. 
We say that allocation $x$ is efficient if there is no allocation $y \in \mathscr{A}$ and no state $\omega^{*} \in \Omega$ such that

$$
\mathrm{E}_{\mu_{i}}\left(u_{i}\left(y_{i}\right) \mid \mathscr{P}_{i}\right)(\omega) \geq \mathrm{E}_{\mu_{i}}\left(u_{i}\left(x_{i}\right) \mid \mathscr{P}_{i}\right)(\omega) \quad \forall \omega \in \mathscr{P}_{N}\left(\omega^{*}\right), \forall i \in N
$$

with strict inequality at $\omega^{*}$ for all $i \in N$.

We say that allocation $x$ is weakly efficient if there is no allocation $y \in \mathscr{A}$ and no state $\omega^{*} \in \Omega$ such that

$$
\mathrm{E}_{\mu_{i}}\left(u_{i}\left(y_{i}\right) \mid \mathscr{P}_{i}\right)(\omega)>\mathrm{E}_{\mu_{i}}\left(u_{i}\left(x_{i}\right) \mid \mathscr{P}_{i}\right)(\omega) \quad \forall \omega \in \mathscr{P}_{N}\left(\omega^{*}\right), \forall i \in N .
$$

A standard argument shows that when preferences are continuous and strictly monotonic at every state of the world the concepts of strict efficiency and efficiency coincide. Weak efficiency, however, is not identical to efficiency even when agents' preferences are continuous and strictly monotonic at every state of the world as the following example demonstrates.

Example 1. Consider the following two-agent two-state economy with a common prior given by $\mu=\left(\frac{1}{2}, \frac{1}{2}\right)$. The agents' utility functions are constant across states and given by $u_{i}(a, b)=a b$, for $i=1,2$. The initial endowments and information partitions are described in the following table, which also contains a feasible allocation $y$.

\begin{tabular}{ccccccc}
\hline \hline & & \multicolumn{2}{c}{ Endowment $e$} & & \multicolumn{2}{c}{ Allocation $y$} \\
\cline { 3 - 4 } \cline { 7 - 8 } Agent & $\mathscr{P}_{i}$ & $\omega_{1}$ & $\omega_{2}$ & & $\omega_{1}$ & $\omega_{2}$ \\
\hline 1 & $\left\{\left\{\omega_{1}\right\},\left\{\omega_{2}\right\}\right\}$ & $(1,1)$ & $(2,0)$ & & $(1,1)$ & $(1,1)$ \\
2 & $\left\{\left\{\omega_{1}, \omega_{2}\right\}\right\}$ & $(0,0)$ & $(0,2)$ & & $(0,0)$ & $(1,1)$ \\
\hline
\end{tabular}

The initial endowment is not an efficient allocation because allocation $y$ dominates it: both agents are better off at state $\omega_{2}$ without being hurt at state $\omega_{1}$. On the other hand the initial endowment is weakly efficient because it is impossible to make agent 1 better off in state $\omega_{1}$.

A sufficient condition for the three efficiency concepts to coincide is that the agents' preferences be continuous and strictly monotonic at every state of the world and that the agents's consumption sets be open subsets of $\mathbb{R}_{++}^{l}$. For in this case, if one agent can be made better off at one state, say $\omega$, without hurting anybody at any state in $\mathscr{P}_{N}(\omega)$, then it is possible to design a system of transfers that makes everybody better off at every sate in $\mathscr{P}_{N}(\omega)$.

The existence of individually rational and strictly efficient allocations is guaranteed if the individuals' utility functions are continuous (see Wilson (1978) for details).

\section{Improvements}

In this section we present several ways - which differ in the degree of communication allowed - a coalition can improve upon an allocation. The first 
notion is due to Wilson (1978) and allows no communication between the agents. In order for a coalition to improve upon an allocation it must be common knowledge among the members of the coalition, with respect to their initial information partitions, that they can enforce an $S$-allocation that is strictly preferred by all of them.

Definition 7. Let $T$ be a coalition, let $x$ be an assignment of bundles to agents in $T$, and let $\left(\Pi_{i}\right)_{i \in T}$ be an information structure for $T$. Let $S \subseteq T$ be another coalition. We say that $S$-allocation $y \in \mathscr{A}(S)$ is a coarse improvement of $S$ upon $x$ with respect to information structure $\left(\Pi_{i}\right)_{i \in T}$ at $\omega^{*}$ if

$$
\mathrm{E}_{\mu_{i}}\left[u_{i}\left(y_{i}\right) \mid \Pi_{i}\right](\omega)>\mathrm{E}_{\mu_{i}}\left[u_{i}\left(x_{i}\right) \mid \Pi_{i}\right](\omega) \quad \forall i \in S, \forall \omega \in \Pi_{S}\left(\omega^{*}\right) .
$$

When $y$ is a coarse improvement of $S$ upon $x$ with respect to $\left(\Pi_{i}\right)_{i \in T}$ at some $\omega \in \Omega$, we say that $x$ is improved upon by $S$ with respect to $\left(\Pi_{i}\right)_{i \in T}$.

Associated with the notion of coarse improvements, we have the definition of the Coarse Core.

Definition 8. The Coarse Core of the economy $\mathscr{E}$ with respect to information structure $\left(\Pi_{i}\right)_{i \in N}$ is the set of its allocations that cannot be improved upon with respect to $\left(\Pi_{i}\right)_{i \in N}$ by any coalition.

The Coarse Core of the economy $\mathscr{E}$ with respect to information structure $\left(\mathscr{P}_{i}\right)_{i \in N}$ is simply called the Coarse Core of $\mathscr{E}$.

The second notion is appropriate when agents have unlimited communication possibilities. By unlimited communication possibilities we mean that each coalition has a set of feasible communication systems at its disposal (see Wilson (1978), Section 3), which is rich enough to enable the coalition to use any of the information structures between the initial one and the coarsest common refinement of the initial one.

Definition 9. Let $\mathscr{E}=\left\langle N,\left(\mathscr{P}_{i}, u_{i}, e_{i}, \mu_{i}\right)_{i \in N}\right\rangle$ be an economy and let $x$ be an allocation in it. We say that $S$-allocation $y$ is a fine improvement of $S$ upon $x$ if there is an information structure $\left(\Pi_{k}\right)_{k \in S}$ for $S$ such that

for all $i \in S, \Pi_{i}$ is not coarser than $\mathscr{P}_{i}$

for all $i \in S, \Pi_{i}$ is not finer than $\bigvee_{k \in S} \mathscr{P}_{k}$

and with respect to which $y$ is a coarse improvement of $S$ upon $x_{S}$.

Associated with fine improvements, we have the notion of the Fine Core, found in Wilson (1978).

Definition 10. The Fine Core of the economy $\mathscr{E}$ is the set of all its allocations that cannot be finely improved upon by any coalition.

We want to introduce a notion of improvement, that allows for some, but not unlimited, communication. To understand our notion, note that in order for an $S$-allocation $y$ to be a coarse improvement upon $x$, it must be common knowledge without communication that $y$ is strictly preferred to $x$ by all agents in $S$. Also, in order for $y$ to be a fine improvement upon $x$, it should 
be possible to redistribute the information initially held by the members of the coalition in such a way that no one learns more than what can be learned by pooling all the information, no one forgets what he knows, and finally, in such a way that makes it common knowledge that $y$ is strictly preferred to $x$. Our notion lies somewhere in the middle since it does not allow unlimited information transmission. We allow only the transmission of information that takes place when the agents repeatedly agree to carry out the transaction. The fact that $y$ is strictly preferred to $x$ by all members of $S$ should become common knowledge after a long handshake during which the agents ratify their willingness to take $y$ instead of $x$. Only after this long handshake can we say that it became common knowledge that $y$ is better than $x$. In order to formalize the idea we need a piece of notation.

Let $f$ and $g$ be two functions on $\Omega$. We denote the event at which $f>g$ by $[f>g]$. Namely,

$$
[f>g]=\{w \in \Omega: f(w)>g(w)\} .
$$

Similarly $[f \leq g]=[f>g]^{c}$. Also, we denote by $\mathscr{P}(f>g)$ the partition generated by $[f>g]$, namely

$$
\mathscr{P}(f>g)=\{[f>g],[f \leq g]\} .
$$

Definition 11. Let $T$ be a coalition, let $x$ be an assignment of bundles to agents in $T$, let $\left(\Pi_{i}\right)_{i \in T}$ be an information structure for $T$, and let $S \subseteq T$ be a coalition. Let $y \in \mathscr{A}(S)$ be an $S$-allocation and define the information structures for $S$, $\left(\Pi_{i}^{S}(n)\right)_{i \in S}$, for $n=0,1,2, \ldots$, recursively as follows:

$$
\Pi_{i}^{S}(0)=\Pi_{i} \quad \forall i \in S,
$$

for $n \in \mathbb{N}$,

$$
\begin{aligned}
& \Pi_{i}^{S}(n+1) \\
& \quad=\Pi_{i}^{S}(n) \vee\left[\bigvee_{k \in S} \mathscr{P}\left(\mathrm{E}_{\mu_{k}}\left(u_{k}\left(y_{k}\right) \mid \Pi_{k}^{S}(n)\right)>\mathrm{E}_{\mu_{k}}\left(u_{k}\left(x_{k}\right) \mid \Pi_{k}^{S}(n)\right)\right)\right] \quad \forall i \in S
\end{aligned}
$$

and

$$
\Pi_{i}^{S}=\lim _{n \rightarrow \infty} \Pi_{i}^{S}(n)
$$
$\Omega$ if

We say that $\left\langle y,\left(\Pi_{i}^{S}\right)_{i \in S}\right\rangle$ is an improvement of $S$ upon $\left\langle x,\left(\Pi_{i}\right)_{i \in T}\right\rangle$ at $\omega \in$

$$
\mathrm{E}_{\mu_{i}}\left[u_{i}\left(y_{i}\right) \mid \Pi_{i}^{S}(n)\right](\omega)>\mathrm{E}_{\mu_{i}}\left[u_{i}\left(x_{i}\right) \mid \Pi_{i}^{S}(n)\right](\omega) \quad \forall i \in S, \forall n \in \mathbb{N} .
$$

When $\left\langle y,\left(\Pi_{i}^{S}\right)_{i \in S}\right\rangle$ is an improvement of $S$ upon $\left\langle x,\left(\Pi_{i}\right)_{i \in N}\right\rangle$ at some $\omega$, we say that $\left\langle x,\left(\Pi_{i}\right)_{i \in N}\right\rangle$ is improved upon by $S$.

Implicit in the above definition is a "dialogue" in the sense of Bacharach (1985). In this dialogue agents repeatedly communicate their willingness to 
perform a trade. In order for $\left\langle y,\left(\Pi_{i}^{S}\right)_{i \in S}\right\rangle$ to be an improvement of $S$ upon $\left\langle x,\left(\Pi_{i}\right)_{i \in N}\right\rangle$ at $\omega$, the following should hold:

1. All agents in $S$ should prefer $y$ to $x$ at $\omega$ when they evaluate these assignments according to the information structure $\Pi_{i}$

2. All agents in $S$ should prefer $y$ to $x$ at $\omega$ when they evaluate these assignments according to the information structure that results from the refinement of $\Pi_{i}$ with the information that all the previous items hold

3. The same as above, and so on ad infinitum.

Note that in order for $y$ to be part of an improvement upon $x$, it must be the case that the agents prefer $y$ to $x$ during the whole dialogue. An alternative definition would require only that the agents prefer $y$ to $x$ in the limit, that is, independently of the answers along the dialogue.

Clearly, since the individual partitions are assumed to be finite, the refinement process just described ends after a finite number of rounds. More formally, by the definition of the partitions $\Pi_{i}^{S}(n)$, the sequence $\left\{\Pi_{i}^{S}(n)\right\}_{n \in \mathbb{N}}$ is non-decreasing. Since the state space is finite, there must be a step $M \in \mathbb{N}$ such that for all $n>M$ we have $\Pi_{i}^{S}(n)=\Pi_{i}^{S}(n+1)$ for all $i \in S$. This shows that the partitions $\Pi_{i}^{S}$ are well-defined and satisfy

$$
\Pi_{i}^{S}=\Pi_{i}^{S} \vee\left[\bigvee_{k \in S} \mathscr{P}\left(\mathrm{E}_{\mu_{k}}\left(u_{k}\left(y_{k}\right) \mid \Pi_{k}^{S}\right)>\mathrm{E}_{\mu_{k}}\left(u_{k}\left(x_{k}\right) \mid \Pi_{k}^{S}\right)\right)\right] .
$$

Remark 1. Assume $\left\langle y,\left(\Pi_{i}^{S}\right)_{i \in S}\right\rangle$ is an improvement of $S$ upon $\left\langle x,\left(\Pi_{i}\right)_{i \in N}\right\rangle$ at some $\omega$, then $y$ is a coarse improvement of $S$ upon $x_{S}$ with respect to the information structure $\left(\Pi_{i}^{S}\right)_{i \in S}$. Namely, it became common knowledge among the members of $S$ that $x$ is strictly preferred to $y$.

Proof: Since $\left\langle y,\left(\Pi_{i}^{S}\right)_{i \in S}\right\rangle$ is an improvement upon $\left\langle x,\left(\Pi_{i}\right)_{i \in S}\right\rangle$ at $\omega$ we have

$$
\mathrm{E}_{\mu_{i}}\left(u_{i}\left(y_{i}\right) \mid \Pi_{i}^{S}(n)\right)(\omega)>\mathrm{E}_{\mu_{i}}\left(u_{i}\left(x_{i}\right) \mid \Pi_{i}^{S}(n)\right)(\omega) \quad \forall i \in S \forall n \in \mathbb{N} .
$$

Since $\Pi_{i}^{S}(n)=\Pi_{i}^{S}$ for some $n \in \mathbb{N}$ we have

$$
\mathrm{E}_{\mu_{i}}\left(u_{i}\left(y_{i}\right) \mid \Pi_{i}^{S}\right)(\omega)>\mathrm{E}_{\mu_{i}}\left(u_{i}\left(x_{i}\right) \mid \Pi_{i}^{S}\right)(\omega) \quad \forall i \in S .
$$

This, together with equality (1), implies that

$$
\Pi_{i}^{S}(\omega) \subseteq \bigcap_{k \in S}\left[\mathrm{E}_{\mu_{k}}\left(u_{k}\left(y_{k}\right) \mid \Pi_{k}^{S}\right)>\mathrm{E}_{\mu_{k}}\left(u_{k}\left(x_{k}\right) \mid \Pi_{k}^{S}\right)\right] \quad \forall i \in S
$$

Consequently,

$$
\Pi_{S}^{S}(\omega) \subseteq \bigcap_{k \in S}\left[\mathrm{E}_{\mu_{k}}\left(u_{k}\left(y_{k}\right) \mid \Pi_{k}^{S}\right)>\mathrm{E}_{\mu_{k}}\left(u_{k}\left(x_{k}\right) \mid \Pi_{k}^{S}\right)\right]
$$

which means that it is common knowledge with respect to $\left(\Pi_{k}^{S}\right)_{k \in S}$ at $\omega$ that all members of $S$ prefer $y$ to $x_{S}$. 
The previous remark allows us to say that if $\left\langle y,\left(\Pi_{i}^{S}\right)_{i \in S}\right\rangle$ is an improvement of $S$ upon $\left\langle x,\left(\Pi_{i}\right)_{i \in N}\right\rangle$ at some $\omega$, then it can become common knowledge among the members of $S$ that $x$ is strictly preferred to $y$.

In order to understand the definition of an improvement, consider the following example.

Example 2. Consider the following three-agent four-state economy with a common prior given by $\mu=\left(\frac{1}{4}, \frac{1}{4}, \frac{1}{4}, \frac{1}{4}\right)$. The agents' utility functions are constant across states and given by $u_{i}(a, b)=a b$, for $i=1,2,3$. The initial endowments and information partitions are described in the following table, which also contains a feasible allocation $y$.

\begin{tabular}{|c|c|c|c|c|c|c|c|c|c|}
\hline \multirow[b]{2}{*}{ Agent } & \multirow[b]{2}{*}{$\mathscr{P}_{i}$} & \multicolumn{4}{|c|}{ Endowment $e$} & \multicolumn{4}{|c|}{ Allocation $y$} \\
\hline & & $\omega_{1}$ & $\omega_{2}$ & $\omega_{3}$ & $\omega_{4}$ & $\omega_{1}$ & $\omega_{2}$ & $\omega_{3}$ & $\omega_{4}$ \\
\hline 1 & $\left\{\left\{\omega_{1}, \omega_{2}, \omega_{3}\right\},\left\{\omega_{4}\right\}\right\}$ & $(2,0)$ & $(1,1)$ & $(0,0)$ & $(1,1)$ & $(1,1)$ & $(1,1)$ & $(0,0)$ & $(0,1)$ \\
\hline 2 & $\left\{\left\{\omega_{1}, \omega_{2}\right\},\left\{\omega_{3}, \omega_{4}\right\}\right\}$ & $(1,0)$ & $(0,0)$ & $(1,1)$ & $(0,1)$ & $(1,1)$ & $(1,1)$ & $(0,0)$ & $(1,2)$ \\
\hline 3 & $\left\{\left\{\omega_{1}\right\},\left\{\omega_{2}, \omega_{3}, \omega_{4}\right\}\right\}$ & $(0,3)$ & $(1,1)$ & $(0,1)$ & $(0,1)$ & $(1,1)$ & $(0,0)$ & $(1,2)$ & $(0,0)$ \\
\hline
\end{tabular}

The expected utilities of each agent, derived from the above allocations, are given by:

\begin{tabular}{lcccc}
\hline \hline & $\omega_{1}$ & $\omega_{2}$ & $\omega_{3}$ & $\omega_{4}$ \\
\hline $\mathrm{E}\left(u_{1}\left(e_{1}\right) \mid \mathscr{P}_{1}\right)$ & $\{1 / 3$ & $1 / 3$ & $1 / 3\}$ & $\{1\}$ \\
$\mathrm{E}\left(u_{1}\left(y_{1}\right) \mid \mathscr{P}_{1}\right)$ & $\{2 / 3$ & $2 / 3$ & $2 / 3\}$ & $\{0\}$ \\
$\mathrm{E}\left(u_{2}\left(e_{2}\right) \mid \mathscr{P}_{2}\right)$ & $\{0$ & $0\}$ & $\{1 / 2$ & $1 / 2\}$ \\
$\mathrm{E}\left(u_{2}\left(y_{2}\right) \mid \mathscr{P}_{2}\right)$ & $\{1$ & $1\}$ & $\{1$ & $1\}$ \\
$\mathrm{E}\left(u_{3}\left(e_{3}\right) \mid \mathscr{P}_{3}\right)$ & $\{0\}$ & $\{1 / 3$ & $1 / 3$ & $1 / 3\}$ \\
$\mathrm{E}\left(u_{3}\left(y_{3}\right) \mid \mathscr{P}_{3}\right)$ & $\{1\}$ & $\{2 / 3$ & $2 / 3$ & $2 / 3\}$ \\
\hline
\end{tabular}

At no state of the world is it common knowledge that all agents prefer the assignment $y$ to the assignment $e$, because at state $\omega_{4}$ agent 1 strictly prefers $x$ to $y$. Consequently, $y$ is not a coarse improvement of $N$ upon the endowment.

Assume, however, that the true state is $\omega_{1}$. At this state all three agents prefer $y$ to $x$. Since this is true for all states except for $\omega_{4}$, it becomes common knowledge, after $y$ has not been rejected by any agent, that state $\omega_{4}$ is not the true state.

This gives rise to the following information structure:

$$
\begin{aligned}
& \Pi_{1}(1)=\left\{\left\{\omega_{1}, \omega_{2}, \omega_{3}\right\},\left\{\omega_{4}\right\}\right\} \\
& \Pi_{2}(1)=\left\{\left\{\omega_{1}, \omega_{2}\right\},\left\{\omega_{3}\right\},\left\{\omega_{4}\right\}\right\} \\
& \Pi_{3}(1)=\left\{\left\{\omega_{1}\right\},\left\{\omega_{2}, \omega_{3}\right\},\left\{\omega_{4}\right\}\right\}
\end{aligned}
$$

and the corresponding new expected utilities for each agent: 


\begin{tabular}{lcccc}
\hline \hline & $\omega_{1}$ & $\omega_{2}$ & $\omega_{3}$ & $\omega_{4}$ \\
\hline $\mathrm{E}\left(u_{1}\left(x_{1}\right) \mid \Pi_{1}(1)\right)$ & $\{1 / 3$ & $1 / 3$ & $1 / 3\}$ & $\{1\}$ \\
$\mathrm{E}\left(u_{1}\left(y_{1}\right) \mid \Pi_{1}(1)\right)$ & $\{2 / 3$ & $2 / 3$ & $2 / 3\}$ & $\{0\}$ \\
$\mathrm{E}\left(u_{2}\left(x_{1}\right) \mid \Pi_{2}(1)\right)$ & $\{0$ & $0\}$ & $\{1\}$ & $\{0\}$ \\
$\mathrm{E}\left(u_{2}\left(y_{2}\right) \mid \Pi_{2}(1)\right)$ & $\{1$ & $1\}$ & $\{0\}$ & $\{2\}$ \\
$\mathrm{E}\left(u_{3}\left(x_{3}\right) \mid \Pi_{3}(1)\right)$ & $\{0\}$ & $\{1 / 2$ & $1 / 2\}$ & $\{0\}$ \\
$\mathrm{E}\left(u_{3}\left(y_{3}\right) \mid \Pi_{3}(1)\right)$ & $\{1\}$ & $\{1$ & $1\}$ & $\{0\}$ \\
\hline
\end{tabular}

Note that all agents prefer $y$ to $x$ except for agent 2 at state $\omega_{3}\left(\omega_{4}\right.$ is irrelevant at this stage, since it became common knowledge that it did not occur). Consequently, it becomes apparent at this stage (do not forget that we are at $\left.\omega_{1}\right)$ that $\omega_{3}$ is not the true state. This gives rise to the following information structure:

$$
\begin{aligned}
& \Pi_{1}(2)=\left\{\left\{\omega_{1}, \omega_{2}\right\},\left\{\omega_{3}\right\},\left\{\omega_{4}\right\}\right\} \\
& \Pi_{2}(2)=\left\{\left\{\omega_{1}, \omega_{2}\right\},\left\{\omega_{3}\right\},\left\{\omega_{4}\right\}\right\} \\
& \Pi_{3}(2)=\left\{\left\{\omega_{1}\right\},\left\{\omega_{2}\right\},\left\{\omega_{3}\right\},\left\{\omega_{4}\right\}\right\}
\end{aligned}
$$

The corresponding expected utilities, with respect to the updated information structure is given by:

\begin{tabular}{lcccc}
\hline \hline & $\omega_{1}$ & $\omega_{2}$ & $\omega_{3}$ & $\omega_{4}$ \\
\hline $\mathrm{E}\left(u_{1}\left(x_{1}\right) \mid \Pi_{1}(2)\right)$ & $\{1 / 2$ & $1 / 2\}$ & $\{0\}$ & $\{1\}$ \\
$\mathrm{E}\left(u_{1}\left(y_{1}\right) \mid \Pi_{1}(2)\right)$ & $\{1$ & $1\}$ & $\{0\}$ & $\{0\}$ \\
$\mathrm{E}\left(u_{2}\left(x_{2}\right) \mid \Pi_{2}(2)\right)$ & $\{0$ & $0\}$ & $\{1\}$ & $\{0\}$ \\
$\mathrm{E}\left(u_{2}\left(y_{2}\right) \mid \Pi_{2}(2)\right)$ & $\{1$ & $1\}$ & $\{0\}$ & $\{2\}$ \\
$\mathrm{E}\left(u_{3}\left(x_{3}\right) \mid \Pi_{3}(2)\right)$ & $\{0\}$ & $\{1\}$ & $\{0\}$ & $\{0\}$ \\
$\mathrm{E}\left(u_{3}\left(y_{3}\right) \mid \Pi_{3}(2)\right)$ & $\{1\}$ & $\{0\}$ & $\{2\}$ & $\{0\}$ \\
\hline
\end{tabular}

At this point it becomes common knowledge that the true state is $\omega_{1}$, because if the true state were $\omega_{2}$, agent 3 would prefer $x$ to $y$, while at $\omega_{1}$ all three agents prefer $y$ to $x$. We can conclude that:

Allocation $y$ together with the limit information structure

$$
\Pi_{i}(3)=\left\{\left\{\omega_{1}\right\},\left\{\omega_{2}\right\},\left\{\omega_{3}\right\},\left\{\omega_{4}\right\}\right\}, \quad i=1,2,3
$$

is an improvement of $N$ upon $\left\langle x,\left(\mathscr{P}_{i}\right)_{i \in N}\right\rangle$.

The above definition of an improvement leads to the following definition of a core with endogenous communication.

Definition 12. The Core with endogenous communication, or simply the Core, of $\mathscr{E}$ is the set of all allocations $x \in \mathscr{A}$, such that $\left\langle x,\left(\mathscr{P}_{i}\right)_{i \in N}\right\rangle$ is not improved upon by any coalition. 
Given the motivation of the Core, the next two propositions can hardly be surprising.

Proposition 1. The core of an economy is a subset of its Coarse Core.

Proof: Assume that $x$ is an allocation that is not in the Coarse Core of the economy. Then there exists an $S$-allocation $y$ and a state $\omega$ at which it is common knowledge among the members of $S$ that $y$ is preferred to $x$ by each of them. But then $\left\langle y,\left(\mathscr{P}_{i}\right)_{i \in S}\right\rangle$ is an improvement of $S$ upon $\left\langle x,\left(\mathscr{P}_{i}\right)_{i \in S}\right\rangle$ at $\omega$, since nothing can be learned from the fact that all agents strictly prefer $y$ to $x$.

Proposition 2. The Fine Core of an economy is a subset of its Core.

Proof: Let $\mathscr{E}$ be an economy with set of agents $N$. Assume that allocation $x$ is not in the Core of $\mathscr{E}$. This means that there is a coalition $S \subseteq N$ with an improvement upon $\left\langle x,\left(\mathscr{P}_{i}\right)_{i \in N}\right\rangle$. Let $\left\langle y,\left(\Pi_{i}^{S}\right)_{i \in S}\right\rangle$ be that improvement. This means that $y$ coarsely improves upon $x_{S}$ with respect to $\left(\Pi_{i}^{S}\right)_{i \in S}$. Moreover, by construction, for all $i \in S, \Pi_{i}^{S}$ is not coarser than $\mathscr{P}_{i}$ and not finer than $\vee_{k \in S} \mathscr{P}_{k}$. Consequently $y$ is a fine improvement upon $x$.

\section{Internally consistent improvements}

A question that has been asked in the context of perfect information is whether the $S$-allocations that are used to improve upon other allocations are themselves immune to deviations of some of the members of $S$. Improvements that are immune to internal deviations are internally consistent. ${ }^{2}$ Ray (1989) showed that in the context of perfect information, it is always possible to find internally consistent improvements upon allocations that are not in the Core. In this section we want to answer the same question but for the context of economies with asymmetric information. As we shall see, Ray's result still holds, as long as there is no communication. As soon as communication is allowed, we'll be able to find allocations outside the core that are not improved upon by any internally consistent improvement.

Definition 13. Let $x \in \mathscr{A}(T)$ be a T-allocation, let $\left(\Pi_{i}\right)_{i \in T}$ be an information structure, and let $S \subseteq T$ be a coalition. We say that $y \in \mathscr{A}(S)$ is an internally consistent coarse improvement of $S$ upon $x$ with respect to $\left(\Pi_{i}\right)_{i \in T}$ at $\omega \in \Omega$

- when $|S|=1$, if $y$ is a coarse improvement upon $x$ with respect to $\left(\Pi_{i}\right)_{i \in T}$ at $\omega$

- when $|S|>1$, if $y$ is a coarse improvement upon $x$ with respect to $\left(\Pi_{i}\right)_{i \in T}$ at $\omega$ such that there is no coalition $F \subsetneq S$ with an internally consistent coarse improvement upon $y$ with respect to $\left(\Pi_{i}\right)_{i \in S}$ at $\omega$.

The following result is the extension of Ray (1989) to economies with asymmetric information.

\footnotetext{
2 The idea of internally consistent improvements is related to the non-cooperative solution concept of Coalition-Proof Nash Equilibrium defined in Bernheim, Peleg, and Whinston (1987).
} 
Theorem 1. Let $\mathscr{E}$ be an economy with asymmetric information, let $x$ be an allocation in it and let $\left(\Pi_{i}\right)_{i \in N}$ be an information structure. The following statements are equivalent:

i. There is a coalition with a coarse improvement upon $x$ with respect to $\left(\Pi_{i}\right)_{i \in N}$

ii. There is a coalition with an internally consistent coarse improvement upon $x$ with respect to $\left(\Pi_{i}\right)_{i \in N}$.

Proof:

$i \rightarrow i$. Assume there is a coalition $S \subseteq N$ with a coarse improvement upon $x$ at some $\omega^{*}$. Namely, there is an $S$-allocation $y$ and event $\Pi_{S}\left(\omega^{*}\right) \in$ $\bigwedge_{k \in S} \Pi_{k}$ such that $\mathrm{E}\left(u_{i}\left(y_{i}\right) \mid \Pi_{i}\right)(\omega)>\mathrm{E}\left(u_{i}\left(x_{i}\right) \mid \Pi_{i}\right)(\omega)$ for all $\omega \in$ $\Pi_{S}\left(\omega^{*}\right)$, for all $i \in S$. If $y$ is an internally consistent coarse improvement of $S$ upon $x$ at $\omega^{*}$, we are done. So assume that there is a coalition $T \subsetneq S$ and an internally consistent coarse improvement $z$ of $T$ upon $y$ at $\omega^{*}$. Namely there is a $T$-allocation $z$ and an event $\Pi_{T}\left(\omega^{*}\right) \in$ $\bigwedge_{k \in T} \Pi_{k}$ such that $\mathrm{E}\left(u_{i}\left(z_{i}\right) \mid \Pi_{i}\right)(\omega)>\mathrm{E}\left(u_{i}\left(y_{i}\right) \mid \Pi_{i}\right)(\omega)$ for all $\omega \in$ $\Pi_{T}\left(\omega^{*}\right)$ and for all $i \in T$. But then, since $\Pi_{T}\left(\omega^{*}\right) \subseteq \Pi_{S}\left(\omega^{*}\right)$, we have that $\mathrm{E}\left(u_{i}\left(z_{i}\right) \mid \Pi_{i}\right)(\omega)>\mathrm{E}\left(u_{i}\left(x_{i}\right) \mid \Pi_{i}\right)(\omega)$ for all $\omega \in \Pi_{T}\left(\omega^{*}\right)$ and for all $i \in T$, which means that $z$ is an internally consistent coarse improvement of $T$ upon $x$ as well.

$i i \rightarrow i$. An internally consistent coarse improvement is, in particular, a coarse improvement.

Next we want to extend the notion of internal consistency to the improvements that define the Core with endogenous communication.

Definition 14. Let $x \in \mathscr{A}(T)$ be a T-allocation, let $\left(\Pi_{i}\right)_{i \in T}$ be an information structure, and let $S \subseteq T$ be a coalition. We say that $\left\langle y,\left(\Pi_{i}^{S}\right)_{i \in S}\right\rangle$ is an internally consistent improvement of $S$ upon $\left\langle x,\left(\Pi_{i}\right)_{i \in T}\right\rangle$ at $\omega \in \Omega$

- when $|S|=1$, if $\left\langle y,\left(\Pi_{i}^{S}\right)_{i \in S}\right\rangle$ is an improvement upon $\left\langle x,(\Pi)_{i \in T}\right\rangle$ at $\omega$

- when $|S|>1$, if $\left\langle y,\left(\Pi_{i}^{S}\right)_{i \in S}\right\rangle$ is an improvement upon $\left\langle x,(\Pi)_{i \in N}\right\rangle$ at $\omega$ such that there is no coalition $F \subseteq S$ with an internally consistent improvement upon $\left\langle y,\left(\Pi_{i}^{S}\right)_{i \in S}\right\rangle$ at $\omega$.

When $\left\langle y,\left(\Pi_{i}^{S}\right)_{i \in S}\right\rangle$ is an internally consistent improvement of $S$ upon $\left\langle x,\left(\Pi_{i}\right)_{i \in N}\right\rangle$ at some $\omega$, we say that $x$ is improved upon by $S$ in an internally consistent way.

Definition 15. The Internally Consistent Core of $\mathscr{E}$ is the set of all allocations $x \in \mathscr{A}$ such that $\left\langle x,\left(\mathscr{P}_{i}\right)_{i \in N}\right\rangle$ is not improved upon by any coalition in an internally consistent way.

The following proposition shows that the previous definition is not superfluous.

Proposition 3. The Core of an economy is a subset of its Internally Consistent Core and the reverse inclusion does not necessarily hold. 
Proof: Since an internally consistent improvement is also an improvement, it is clear that the Core is a subset of the Internally Consistent Core. To see that the reverse inclusion does not necessarily hold, consider the following economy, taken from Wilson (1978). There are three agents, each with the same constant across states utility function, given by $u(a)=\sqrt{a}$. There is a common prior given by the uniform distribution over the states space. Consider allocation $x$ in the next table

\begin{tabular}{|c|c|c|c|c|c|c|c|c|}
\hline \multirow[b]{2}{*}{ Agent } & \multirow[b]{2}{*}{$\mathscr{P}_{i}$} & \multirow[b]{2}{*}{ State $(\omega)$} & \multicolumn{3}{|c|}{ Endowment $(e)$} & \multicolumn{3}{|c|}{ Allocation $(x)$} \\
\hline & & & $\omega_{1}$ & $\omega_{2}$ & $\omega_{3}$ & $\omega_{1}$ & $\omega_{2}$ & $\omega_{3}$ \\
\hline 1 & $\left\{\left\{\omega_{1}\right\},\left\{\omega_{2}, \omega_{3}\right\}\right\}$ & & 5 & 1 & 3 & 5 & 2 & 2 \\
\hline 2 & $\left\{\left\{\omega_{2}\right\},\left\{\omega_{1}, \omega_{3}\right\}\right\}$ & & 3 & 5 & 1 & 2 & 5 & 2 \\
\hline 3 & $\left\{\left\{\omega_{3}\right\},\left\{\omega_{1}, \omega_{2}\right\}\right\}$ & & 1 & 3 & 5 & 2 & 2 & 5 \\
\hline
\end{tabular}

Allocation $x$ is not in the Core because coalition $S=\{1,2\}$ has an improvement at $\omega_{1}$ which is given by the $S$-allocation $y$ and the information structure $\left(\Pi_{i}^{S}\right)_{i \in S}$ below:

\begin{tabular}{cccccc}
\hline \hline & & & \multicolumn{3}{c}{ Allocation $(y)$} \\
\cline { 3 - 6 } Agent & $\Pi_{i}^{S}$ & State & $\omega_{1}$ & $\omega_{2}$ & $\omega_{3}$ \\
\hline 1 & $\left\{\left\{\omega_{1}\right\},\left\{\omega_{2}, \omega_{3}\right\}\right\}$ & & 5.5 & 0 & 0 \\
2 & $\left\{\left\{\omega_{2}\right\},\left\{\omega_{1}\right\},\left\{\omega_{3}\right\}\right\}$ & & 2.5 & 6 & 4 \\
\hline
\end{tabular}

The improvement $\left\langle y,\left(\Pi_{i}^{S}\right)_{i \in S}\right\rangle$ is not internally consistent since it is improved upon by agent 2 at $\omega_{1}$ in an internally consistent way by means of his own endowment.

The same kind of argument can be made for every improvement upon $\left\langle x,\left(\mathscr{P}_{i}\right)_{i \in N}\right\rangle$. This shows that $x$ belongs to the Internally Consistent Core of the economy. ${ }^{3}$

The above example gives evidence of the superiority of the Internally Consistent Core over the Core. The economy has an empty Core, but the improvements that disqualify allocation $x$ are not credible. In any case, the previous result raises the question of what is the appropriate concept of the core when communication is allowed. ${ }^{4}$ Fortunately, however, the basic concepts of individual rationality and efficiency are not seriously influenced by the possibility of communication as the following propositions show.

\footnotetext{
3 Note that allocation $x$ belongs to the Coarse Core too.

4 The fact that allocations outside the Core are not improved upon in an internally consistent way is not due to special manner the information is transmitted. It follows from the fact that there is communication.
} 
Proposition 4. The following statements are equivalent:

i. Allocation $x$ is individually rational

ii. There is no agent with an improvement upon $x$

iii. There is no agent with an internally consistent improvement upon $x$.

Proof: Trivial

Proposition 5. The following statements hold for all allocations $x$ :

i. If $x$ is efficient then the grand coalition does not have an improvement upon $x$;

ii. If the grand coalition does not have an improvement upon $x$ then the grand coalition does not have an internally consistent improvement upon $x$;

iii. If the grand coalition does not have an improvement upon $x$ then $x$ is weakly efficient.

Proof:

i. If $\left\langle y,\left(\Pi_{i}\right)_{i \in N}\right\rangle$ is an improvement of $N$ upon $\left\langle x,\left(\mathscr{P}_{i}\right)_{i \in N}\right\rangle$, then by Remark 1 there is some information structure $\left(\Pi_{i}\right)_{i \in N}$, finer than $\left(\mathscr{P}_{i}\right)_{i \in N}$, with respect to which it becomes common knowledge at some $\omega^{*}$ that everybody prefers $y$ to $x$. In other words,

$$
\mathrm{E}_{\mu_{i}}\left(u_{i}\left(y_{i}\right) \mid \Pi_{i}\right)(\omega)>\mathrm{E}_{\mu_{i}}\left(u_{i}\left(x_{i}\right) \mid \Pi_{i}\right)(\omega) \quad \forall i \in N \forall \omega \in \Pi_{N}\left(\omega^{*}\right) .
$$

Define the allocation $z$ by

$$
z_{i}(\omega)= \begin{cases}y_{i}(\omega) & \text { if } \omega \in \Pi_{N}\left(\omega^{*}\right) \\ x_{i}(\omega) & \text { otherwise }\end{cases}
$$

It is not difficult to see that $z$ dominates $x$ and as a result $x$ cannot be efficient.

ii. If there is no improvement, you will never find an internally consistent one.

iii. Assume that $x$ is not a weakly efficient allocation. Then there is an allocation $y$ and state $\omega^{*}$ at which it is common knowledge that $y$ is better than $x$ in the eyes of all the agents. But this implies that $y$ is an improvement of the grand coalition upon $x$.

Since when the agents' utility functions are continuous and strictly increasing at every state of the world, and when their consumption sets are open subsets of $\mathbb{R}_{++}^{l}$ the concepts of efficiency and weak efficiency are equivalent, it follows from Proposition 5 that an allocation is efficient if and only if the grand coalition does not have an improvement upon it. In other words, in this case the standard concept of efficiency remains valid even after enabling agents to communicate. 
Corollary 1. Let $\mathscr{E}$ be a two person economy where agents have continuous utility functions. The Internally Consistent Core is a nonempty subset of the Coarse Core.

Proof: It follows from Propositions 4 and 5 and from the existence of individually rational and efficient allocations.

Corollary 2. (Modified No-Trade Theorem) Assume $x$ is an efficient allocation. Then there is no allocation $y$ that can become commonly known to be strictly preferred to $x$ by all agents.

Proof: Since $x$ is efficient, by Proposition 5 there is no allocation $y$ and state $\omega$ at which it can become common knowledge that $y$ is preferred to $x$ by all agents.

The well-known No-Trade Theorem (see Milgrom and Stokey (1982), Holmstrom and Myerson (1983) or Rubinstein and Wolinsky (1990) for different versions) states that if an allocation is ex-ante efficient, it must be (interim) efficient as well. The interpretation is that if the agents traded in contingent commodities before they get their private information, and they reached an ex-ante efficient allocation, then after they get their private information, from wherever it may come, it is impossible to find a reallocation that is commonly known to be preferred by all agents. Our modified no-trade theorem is different in that the starting point is a stage at which the individuals already got their private information and in that some information transmission is allowed. Our theorem says that if the allocation is (interim) efficient (and not necessarily ex-ante efficient), it is impossible for the agents to find an allocation that, even after some dialogue takes place, becomes common knowledge to be strictly preferred to the former. Not only is new information incapable of inducing trade, but endogenous communication between the asymmetrically informed agents is incapable as well.

Corollary 1 shows that for two-person economies, the Internally Consistent Core is a subset of the Coarse Core. In general, however, there is no inclusion relation whatsoever, as the following example shows.

Example 3. Consider the following economy with three agents, two commodities, four states of the world, and a common prior $\mu=\left(\frac{1}{4}, \frac{1}{4}, \frac{1}{4}, \frac{1}{4}\right)$. Agents' utility functions are constant across states and given by $u_{i}(a, b)=\sqrt{a+b}$ for both $i=1,2$ and $u_{3}(a, b)=\sqrt{\min \{a, b\}}$.

\begin{tabular}{cccccc}
\hline \hline & & \multicolumn{4}{c}{ Endowment } \\
\cline { 3 - 6 } Agent & $\mathscr{P}_{i}$ & $\omega_{1}$ & $\omega_{2}$ & $\omega_{3}$ & $\omega_{4}$ \\
\hline \multirow{2}{*}{1} & $\left\{\left\{\omega_{1}, \omega_{4}\right\},\left\{\omega_{2}, \omega_{3}\right\}\right\}$ & $(5,0)$ & $(7,0)$ & $(5,0)$ & $(7,0)$ \\
2 & $\left\{\left\{\omega_{1}, \omega_{2}\right\},\left\{\omega_{3}, \omega_{4}\right\}\right\}$ & $(0,5)$ & $(0,7)$ & $(0,5)$ & $(0,7)$ \\
3 & $\left\{\left\{\omega_{1}, \omega_{2}, \omega_{3}, \omega_{4}\right\}\right\}$ & $(2,2)$ & $(0,0)$ & $(2,2)$ & $(0,0)$ \\
\hline
\end{tabular}

The endowment $e$ is not in the Coarse Core because the allocation $y$ below is commonly known (at every state) to be strictly preferred to $e$ by all agents. 


\begin{tabular}{cccccc}
\hline \hline & & \multicolumn{4}{c}{ Allocation $y$} \\
\cline { 3 - 6 } Agent & $\mathscr{P}_{i}$ & $\omega_{1}$ & $\omega_{2}$ & $\omega_{3}$ & $\omega_{4}$ \\
\hline 1 & $\left\{\left\{\omega_{1}, \omega_{4}\right\},\left\{\omega_{2}, \omega_{3}\right\}\right\}$ & $(6,0)$ & $(6,0)$ & $(6,0)$ & $(6,0)$ \\
2 & $\left\{\left\{\omega_{1}, \omega_{2}\right\},\left\{\omega_{3}, \omega_{4}\right\}\right\}$ & $(0,6)$ & $(0,6)$ & $(0,6)$ & $(0,6)$ \\
3 & $\left\{\left\{\omega_{1}, \omega_{2}, \omega_{3}, \omega_{4}\right\}\right\}$ & $(1,1)$ & $(1,1)$ & $(1,1)$ & $(1,1)$ \\
\hline
\end{tabular}

Moreover, it can be checked that $y$ belongs to the Coarse Core. Allocation $y$, however, does not form part of an internally consistent improvement upon $e$ because coalition $S=\{1,2\}$ has the following internally consistent improvement upon it at $\omega_{2}$.

\begin{tabular}{cccccc}
\hline \hline & & \multicolumn{4}{c}{ Allocation $z$} \\
\cline { 3 - 6 } Agent & $\Pi_{i}^{S}$ & $\omega_{1}$ & $\omega_{2}$ & $\omega_{3}$ & $\omega_{4}$ \\
\hline \multirow{2}{*}{1} & $\left\{\left\{\omega_{1}\right\},\left\{\omega_{2}\right\},\left\{\omega_{3}\right\},\left\{\omega_{4}\right\}\right\}$ & $(3,0)$ & $(7,0)$ & $(5,2)$ & $(7,0)$ \\
2 & $\left\{\left\{\omega_{1}\right\},\left\{\omega_{2}\right\},\left\{\omega_{3}\right\},\left\{\omega_{4}\right\}\right\}$ & $(2,5)$ & $(0,7)$ & $(0,3)$ & $(0,7)$ \\
\hline
\end{tabular}

To see this, note that if $\omega_{2}$ is the true state of the world, both agents prefer $z$ to $y$ ( $z$ provides them with an expected utility of 7$)$. Since $\omega_{2}$ is the only state at which both agents prefer $z$ to $y$, they learn from their mutual agreement that the state is indeed $\omega_{2}$, and it becomes common knowledge that $z$ is preferred to $y$ by both of them. Moreover, $z$ is a "credible" $S$-allocation because it cannot be improved upon by any singleton at $\omega_{2}$. What happens is that, even though allocation $y$ is very attractive, agent 3 fears that at $\omega_{2}$ it will become common knowledge between agents 1 and 2 that they can run away with 7 units each, leaving agent 3 in the cold. This justified fear is what destabilizes allocation $y$. Agent 3 does not give in to the alluring powers of allocation $y$ and rejects it.

Consequently, $y$ is not in the Internally Consistent Core. This shows that the Coarse Core is not a subset of the Internally Consistent Core. On the other hand, allocation $z$ does not form part of an internally consistent improvement upon $e$. Moreover, the initial endowment belongs to the Internally Consistent Core. To see this, note that no pair of agents that contains agent 3 can improve upon the endowment since no agent can individually increase agent's 3 utility. It is not difficult to check that agents 1 and 2 cannot find an improvement upon the endowment. And finally all the improvements of the grand coalition are not internally consistent because an internally consistent improvement upon them similar to the one in the example can be found. This shows that the Internally Consistent Core is not a subset of the Coarse Core.

\section{References}

Aumann RJ (1976) Agreeing to disagree. Annals of Statistics 4:1236-1239

Bacharach M (1985) Some extensions to a claim of Aumann in an axiomatic model of knowledge. Journal of Economic Theory 37:167-190

Bernheim BD, Peleg B, Whinston M (1987) Coalition-proof Nash equilibria I: Concepts. Journal of Economic Theory 42:1-12 
Dutta B, Vohra R (1997) Incomplete information, credibility and the core. Unpublished manuscript: Department of Economics, Brown University

Geanakoplos J, Polemarchakis H (1982) We can't disagree forever. Journal of Economic Theory 28:192-200

Hart S, Tauman Y (1996, December) Market crashes without external shocks. Discussion Paper 124, Center for Rationality and Interactive Decision Theory

Holmstrom B, Myerson R (1983) Efficient and durable decision rules with incomplete information. Econometrica 51(6):1799-1819

Krasa S (1999) Unimprovable allocations in economies with incomplete information. Journal of Economic Theory 87:144-168

Milgrom P, Stokey N (1982) Information, trade and common knowledge. Journal of Economic Theory 26:17-27

Ray D (1989) Credible coalitions and the core. International Journal of Game Theory 18(2):185187

Rubinstein A, Wolinsky A (1990) On the logic of "agreeing to disagree" type results. Journal of Economic Theory 51:184-93

Sebenius JK, Geanakoplos J (1983) Don't bet on it: Contingent agreements with asymmetric information. Journal of the American Statistical Association 78(382):424-426

Vohra R (1999) Incomplete information, incentive compatibility and the core. Journal of Economic Theory 86:123-147

Wilson R (1978) Information, efficiency, and the core of an economy. Econometrica 46(4):807816 